\title{
Jig-Shape Optimization of a Low-Boom Supersonic Aircraft
}

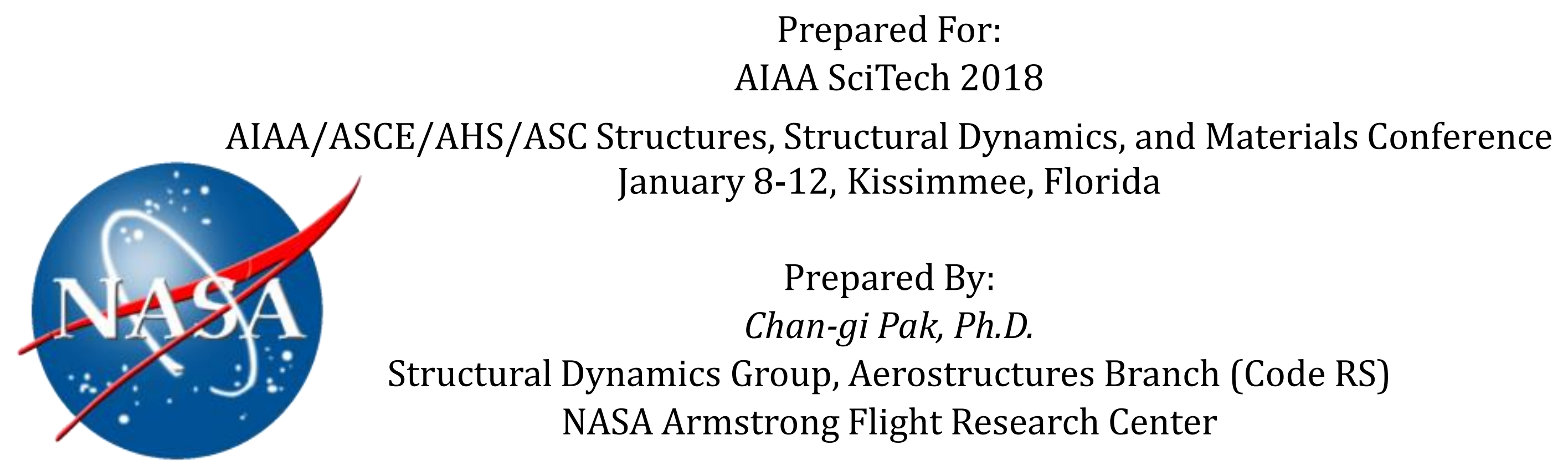




\section{Overview}

$\square$ Theoretical background (slides 3-10)

$\square$ Computational validation (slides 11-28)

$\square$ Conclusions (slide 29) 


\section{Theoretical background}


प Supersonic Commercial Transport Aircraft Design

* Safety

$>$ Light weight airframe can cause strength, buckling, aeroelastic, and aeroservoelastic issues.

* Sonic boom

> Supersonic flight of "commercial transport" aircraft allowed only over the ocean.

$>$ Perceived Loudness in decibels

$\checkmark$ NASA's N+2 goal: 75 PLdB

$\checkmark$ Concorde: 104 PLdB

$\checkmark$ High Speed Civil Transport (HSCT): 99 PLdB

* Fuel efficiency

$>$ Light weight airframe

$>$ Reduced drag

a Developing Low Boom Flight Demonstrator (LBFD)

* Lockheed Martin Skunk Works was the prime contractor for preliminary design of X-plane.

* Loudness: 74 PLdB

ㅁ Major Issue

* Outer-mold-line configuration of an aircraft is design for the desired aerodynamic performance. Assume rigid structure.

- Flexibility of the structure changes the aerodynamic performance.

* It has been reported that one degree of the tip twist of a supersonic wing and stabilator under the cruise flight condition can increase the sonic boom level by 0.2 PLdB and 1.3 PLdB, respectively.

Trim deflection

$$
\left\{\begin{array}{l}
\text { Trimic } \\
\end{array}\right.
$$

Flow
HSCT

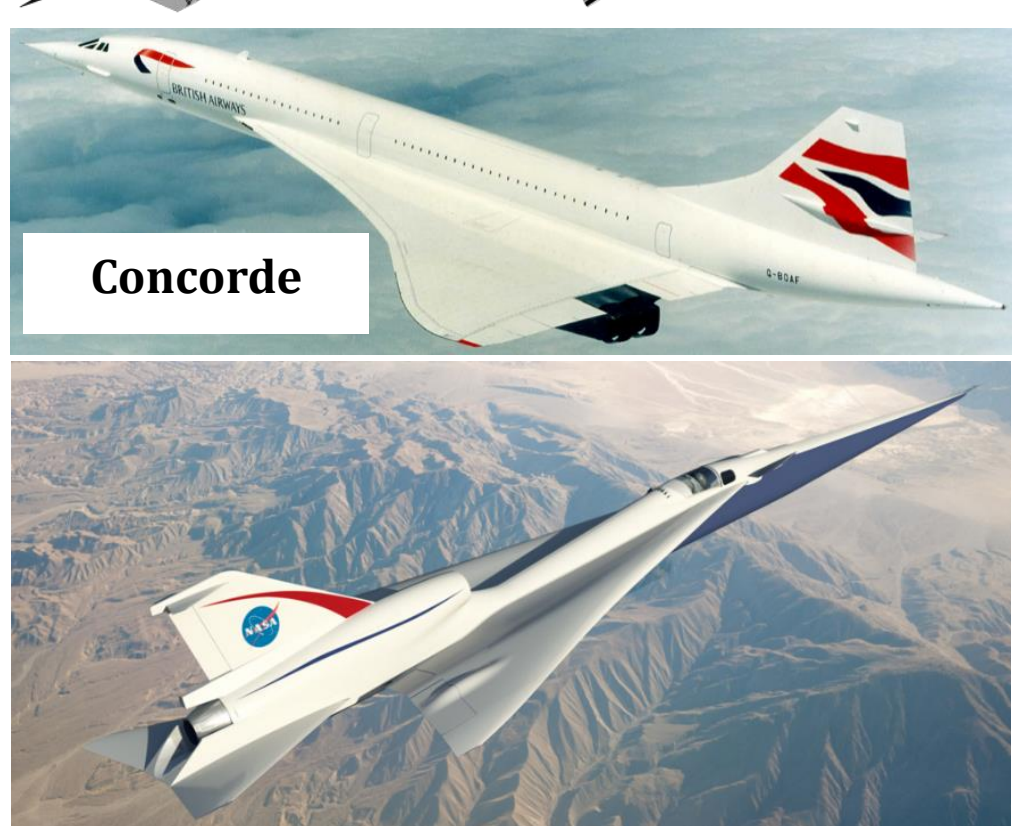

Low boom supersonic aircraft

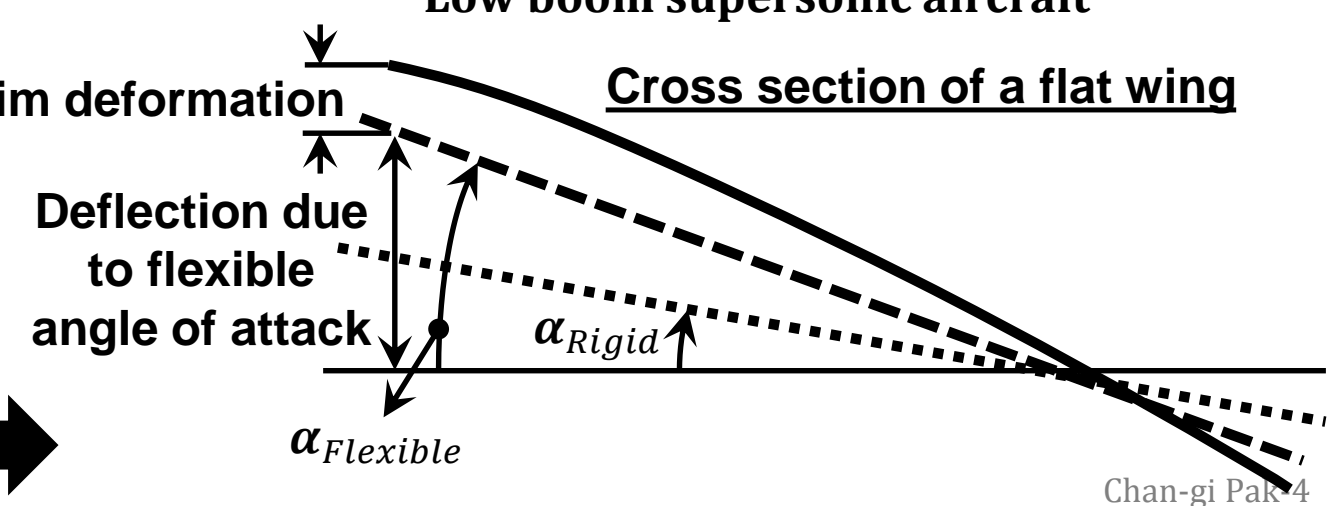




\section{Jig-Shape Optimization Problem Statement}

Assume unconstrained Optimization

Optimization Problem Statement

* Find design variables: $\{X\}=\left[X_{1}, X_{2}, \ldots, X_{n d v}\right\rfloor^{T}$ which

minimize $\left\{F(X)=\sum_{j=1}^{n s u r f \times 3} \Delta T_{j}^{2}\right\}$

* $\{\Delta T\} \equiv\{T\}_{t}-\{T\}_{d}$

- $\{T\}_{t}=$ target trim shape at surface GRIDs

$>\quad$ Sonic boom level is computed based on target trim shape.

* $\quad\{T\}_{d}=$ trim shape based on design jig shape

$>\{j i g\}_{d} \underset{\text { trim analysis }}{\longrightarrow}\{T\}_{d}$

$>\{j i g\}_{d} \equiv\{j i g\}_{b}+\{\Delta j i g\}$

$\checkmark \quad\{j i g\}_{d}=$ design jig-shape

$\checkmark \quad\{j i g\}_{b}=$ baseline jig-shape

$\checkmark \quad\{\Delta j i g\}=$ jig-shape changes

$>\quad\{\Delta j i g\}=[\boldsymbol{\Phi}]\{X\}$

$\checkmark \quad X_{i}=\mathrm{i}$-th design variable

$\checkmark \quad[\Phi]=\left[\{\phi\}_{1}\{\phi\}_{2} \ldots\{\phi\}_{n d v}\right]$

- $\{\phi\}_{i}=\mathrm{i}$-th basis function

- Eigen vector based on jig shape

- Eigen vectors are normalized as Max deflection $=1$ inch 


\section{Update Jig-Shape Module: using shape_change.exe}

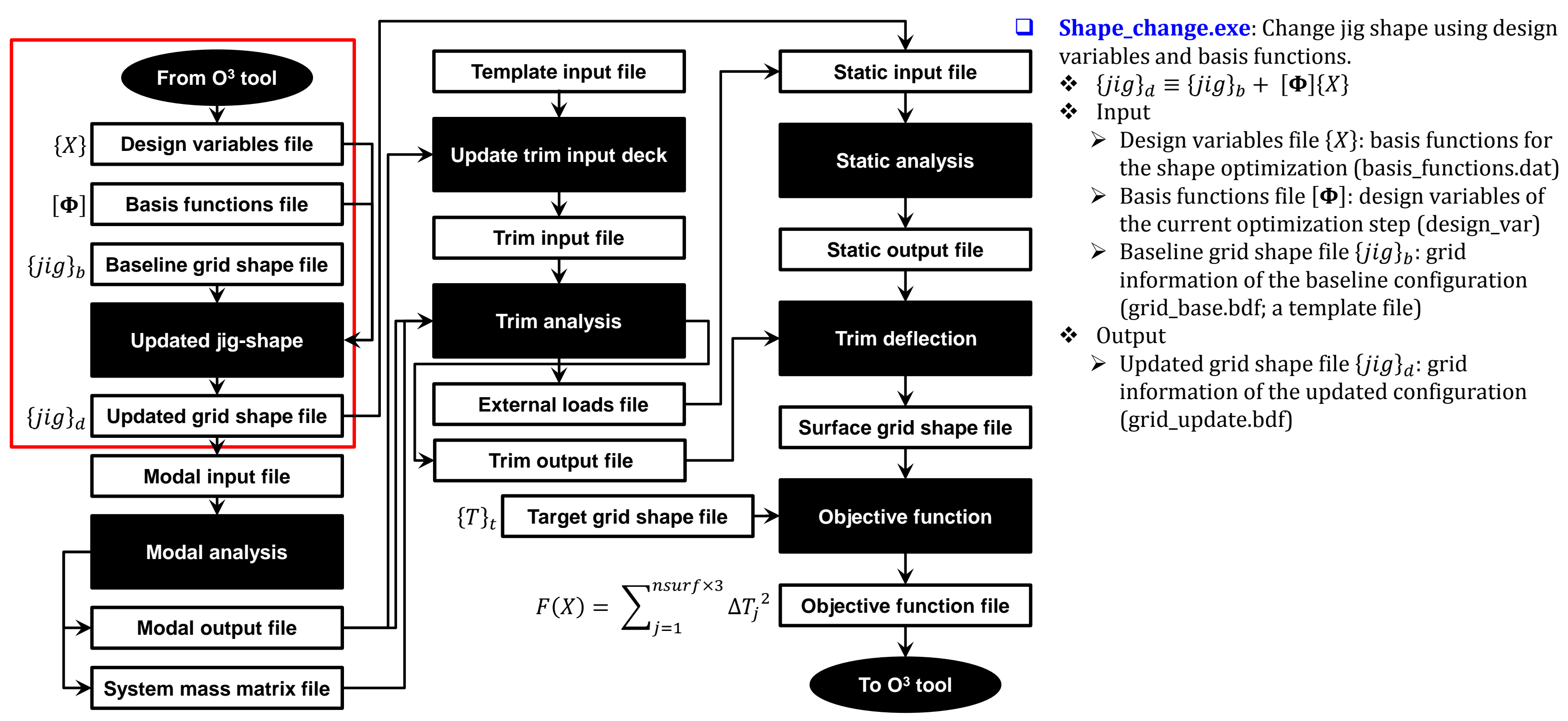

Change jig shape using design variables $\&$ basis functions. 


\section{Modal Analysis Module: using MSC/NASTRAN solution 103}

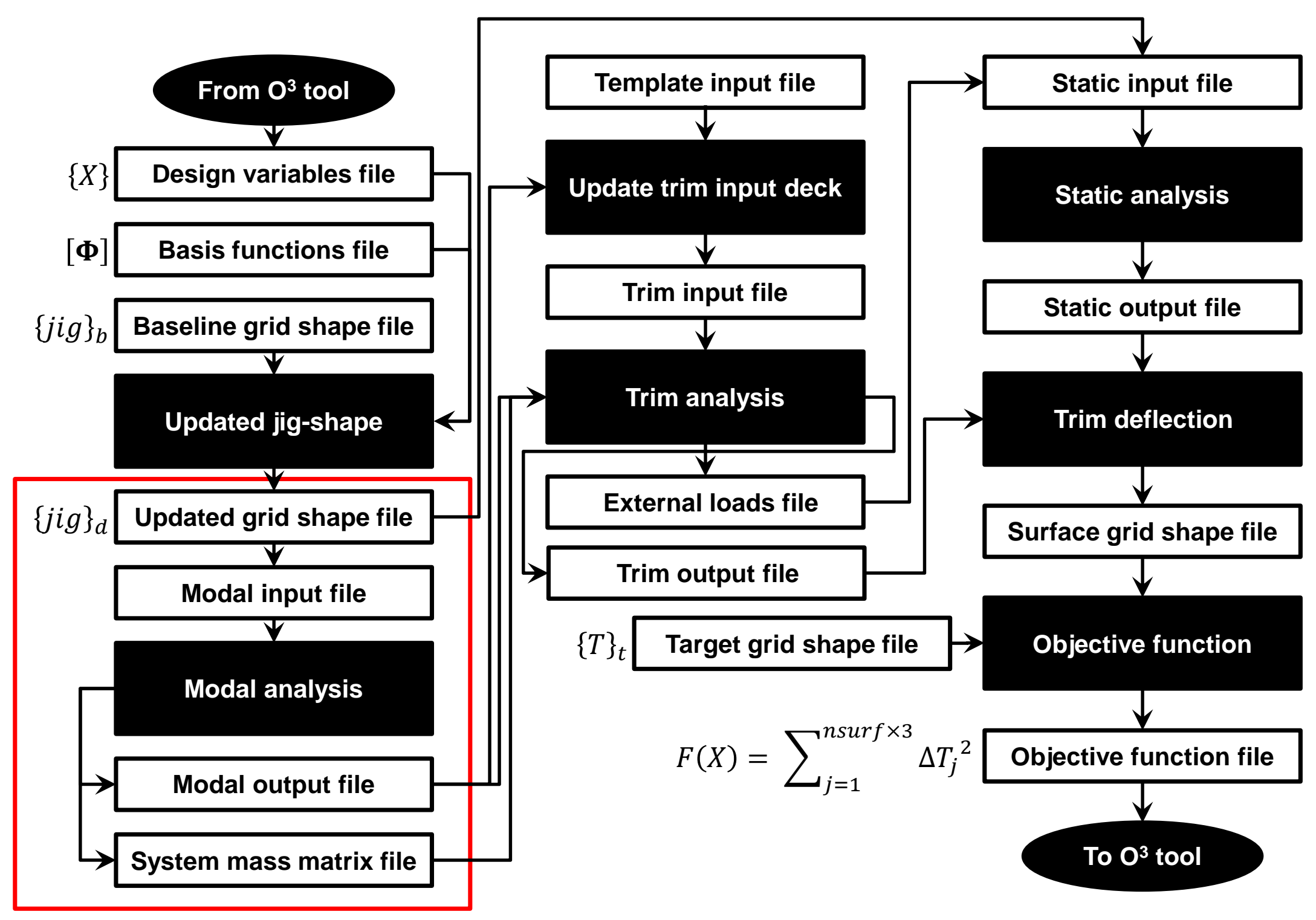

a Perform modal analysis using MSC/NASTRAN solution 103 to change system mass matrix file (MGH matrix), weight, moment of inertia, and CG location for trim analysis.

- Compute six rigid body modes. 


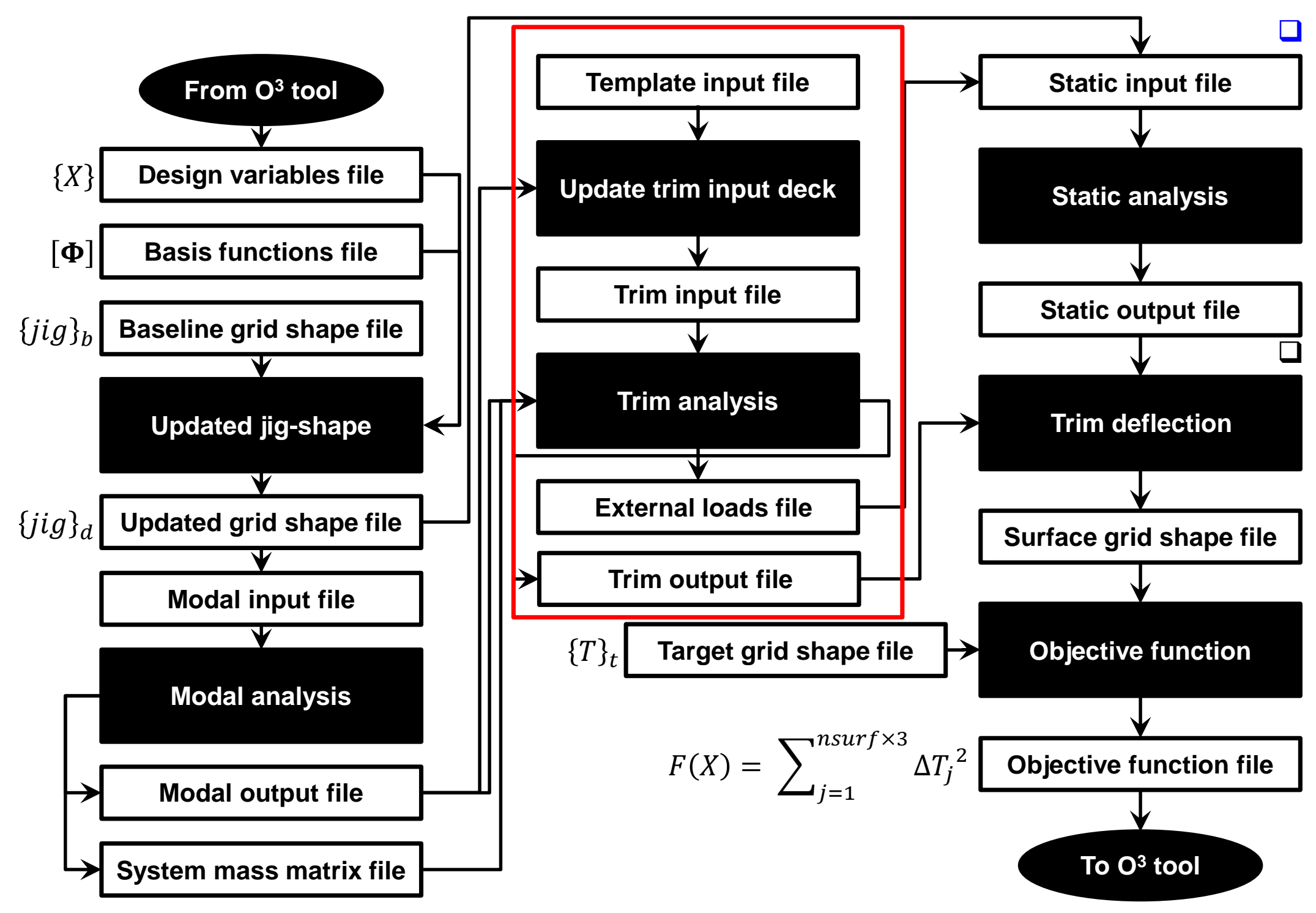

Change_trim.exe: Update input deck for ZAERO trim analysis.

* Input

$>$ Template input file: file for ZAERO based trim analysis (Lbfd_trim.bdf)

Modal output file: f06 file from MSC/NASTRAN * Output

$>$ Trim input file: updated ZAERO input file to be used for trim analysis (trim.bdf)

Perform trim analysis using ZAERO

* Input

$>$ Trim input file: Trim.bdf

* Output

$>$ External loads file: aerodynamic load + inertial load (Extload.dat)

$>$ Trim output file: ZAERO output (trim results) 


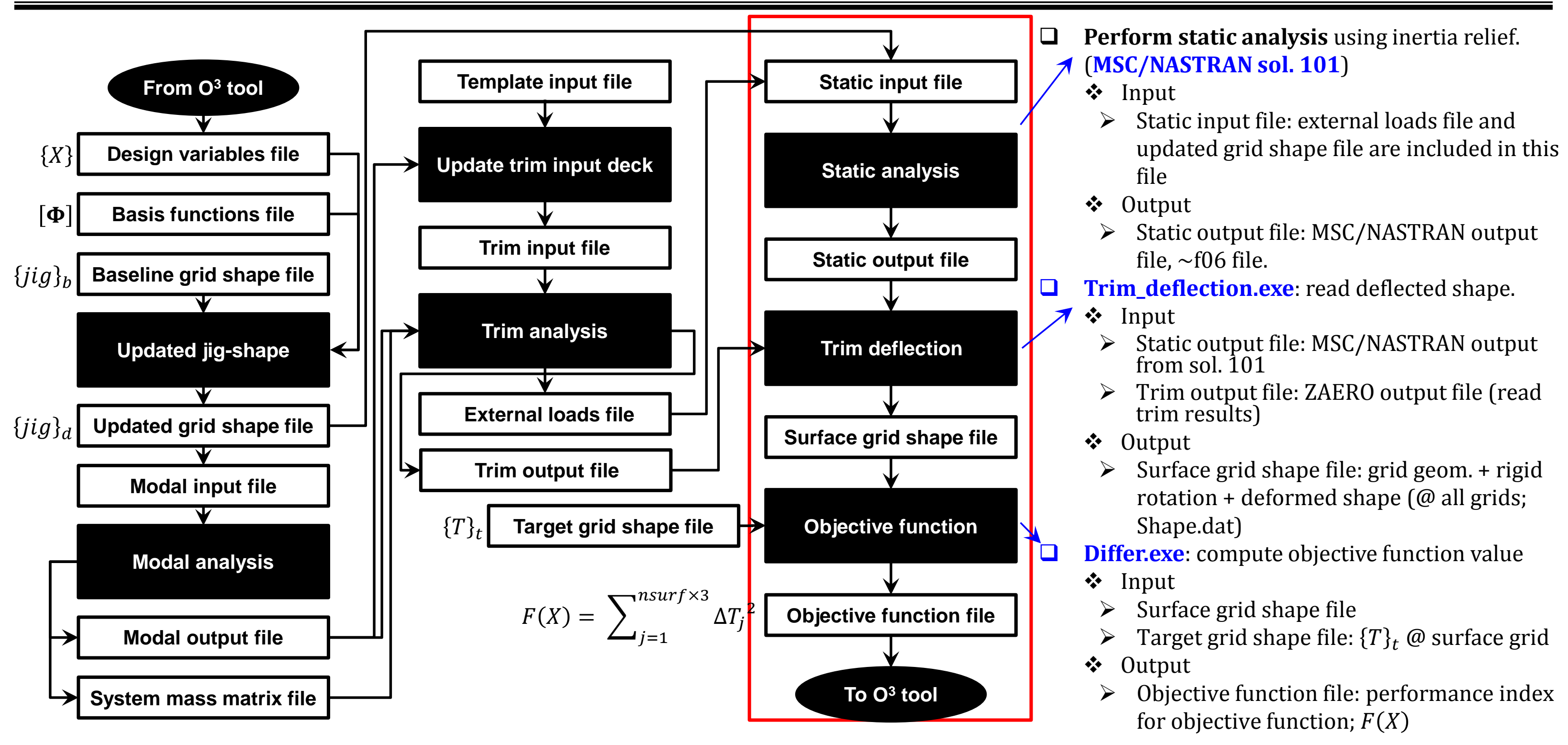




\section{Compute Starting Design Variables: Using Least Squares Surface Fitting Technique}

口 $\{\Delta T\}_{t} \equiv\{T\}_{t}-\{T\}_{b}$

* $\{T\}_{t}=$ target trim shape at surface GRIDs

* $\{T\}_{b}=$ trim shape based on the baseline jig-shape

$>\{j i g\}_{b} \underset{\overline{\text { trim analysis }}}{\Longrightarrow}\{T\}_{b}$

- Fitting $\{\Delta T\}_{t}$ surface using perturbed shapes $\{\Delta T\}_{i}, i=1,2, \ldots, n d v$

* Perturb baseline jig-shape using basis functions [\$]

$>\{j i g\}_{d} \equiv\{j i g\}_{b}+[\boldsymbol{\Phi}]\{X\}$

$>$ Where, $\{\phi\}_{i}=\mathrm{i}$-th basis function

$>\{j i g\}_{b}+\{\phi\}_{i} \underset{\overline{\text { trim analysis }}}{\longrightarrow}\{T\}_{i}$

$>\{\Delta T\}_{i} \equiv\{T\}_{i}-\{T\}_{b}$ (i-th perturbed shape)

- Define a matrix: $[\boldsymbol{\Psi}]=\left[\{\Delta T\}_{1}\{\Delta T\}_{2} \ldots\{\Delta T\}_{n d v}\right]$

ㅁ $[\Psi]\{X\}=\{\Delta T\}_{t}$

* $[\boldsymbol{\Psi}]^{T}[\boldsymbol{\Psi}]\{X\}=[\boldsymbol{\Psi}]^{T}\{\Delta T\}_{t}$

* $\left([\boldsymbol{\Psi}]^{T}[\boldsymbol{\Psi}]\right)^{-1}[\boldsymbol{\Psi}]^{T}[\boldsymbol{\Psi}]\{X\}=\left([\boldsymbol{\Psi}]^{T}[\boldsymbol{\Psi}]\right)^{-1}[\boldsymbol{\Psi}]^{T}\{\Delta T\}_{t}$

Starting design variables: $\{X\}=\left([\boldsymbol{\Psi}]^{T}[\Psi]\right)^{-1}[\Psi]^{T}\{\Delta T\}_{t}$

\section{$\{\Delta T\}_{t}$}
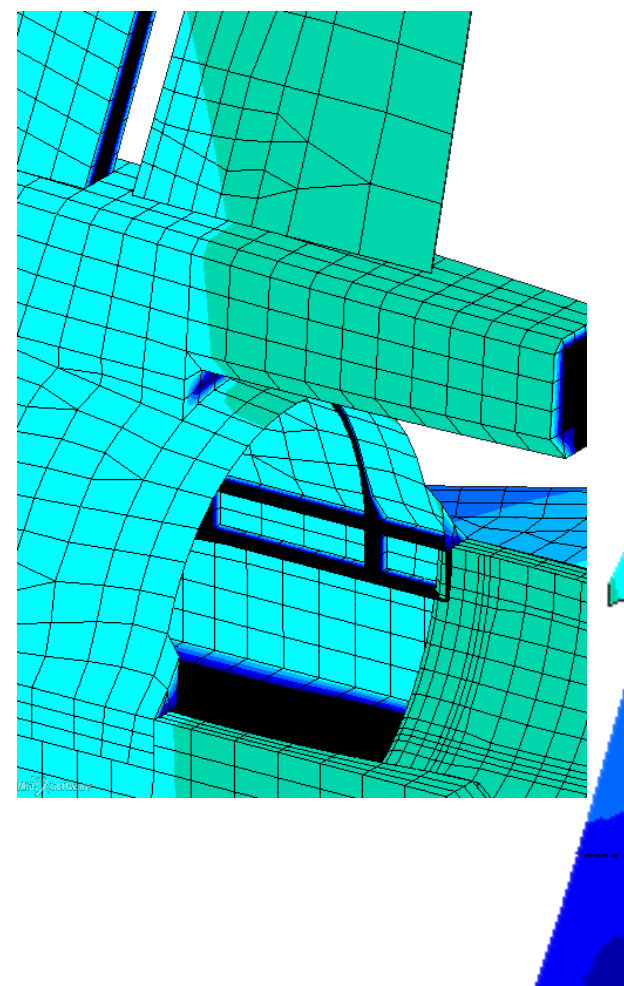

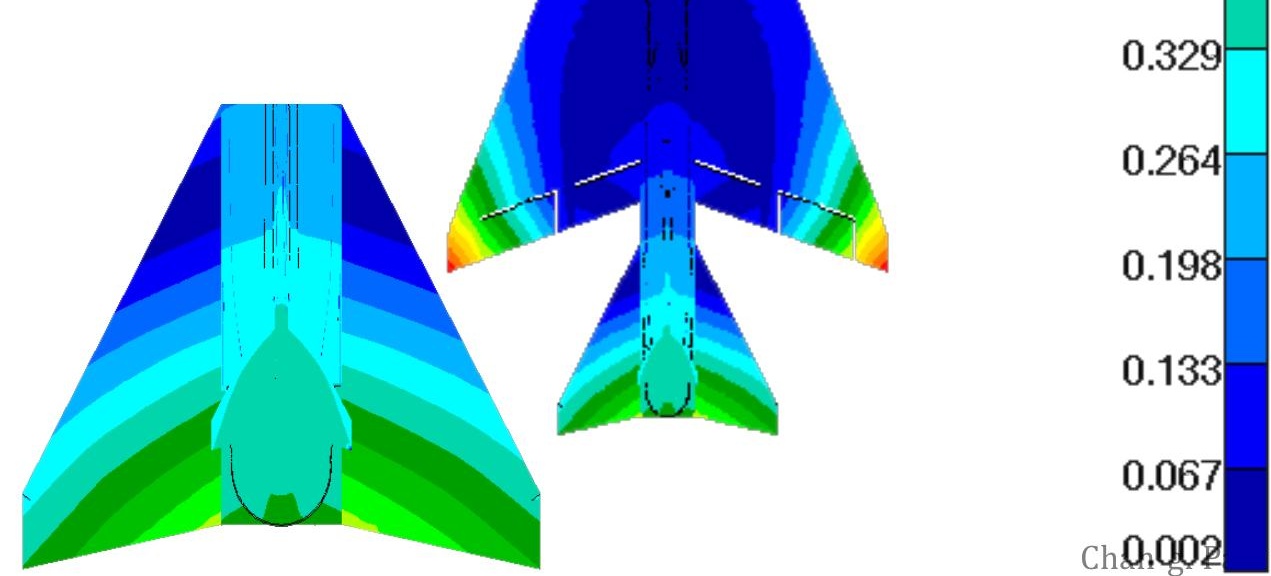




\section{Computational validation}




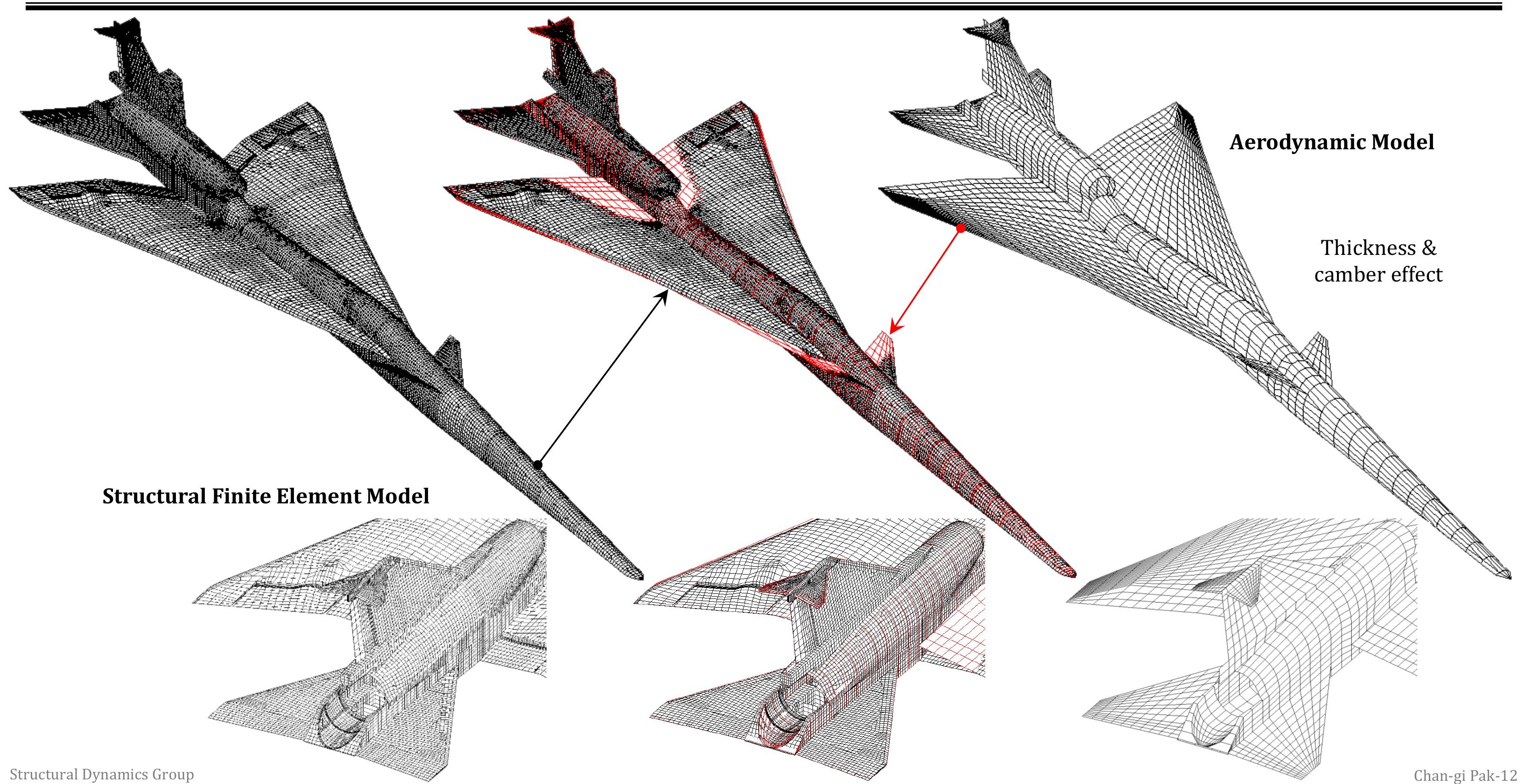




\begin{tabular}{|c|c|c|c|c|}
\hline \multirow{2}{*}{ Mode } & \multicolumn{3}{|c|}{ Frequency (Hz) } & \multirow{2}{*}{ Notes } \\
\hline & Baseline & Optimum & $\begin{array}{c}\% \\
\text { difference }\end{array}$ & \\
\hline 9 & 9.045 & 9.032 & -0.14 & First wing bending + forward fuselage vertical bending + stabilator rotation \\
\hline 11 & 11.97 & 11.97 & 0.00 & Forward fuselage vertical bending + first wing bending + stabilator rotation (Asymmetric) \\
\hline 15 & 14.76 & 14.76 & 0.00 & Stabilator rotation \\
\hline 17 & 19.23 & 19.22 & -0.05 & Wing tip bending + T-tail rotation + flap bending (Asymmetric) \\
\hline 19 & 20.08 & 20.08 & 0.00 & T-tail rotation (Asymmetric) \\
\hline 20 & 20.54 & 20.55 & 0.05 & $\begin{array}{l}\text { Wing tip bending }+ \text { T-tail rotation }+ \text { aileron rotation }+ \text { flap bending }+ \text { forward fuselage vertical bending } \\
\text { (Asymmetric) }\end{array}$ \\
\hline 22 & 21.75 & 21.76 & 0.05 & Aileron rotation + flap rotation $+\mathrm{T}$-tail bending + outboard wing bending torsion \\
\hline 25 & 22.70 & 22.70 & 0.00 & Flap rotation + aileron rotation + T-tail bending (Asymmetric) \\
\hline 37 & 30.79 & 30.75 & -0.13 & Canard bending \\
\hline 48 & 42.96 & 42.97 & 0.02 & T-tail bending (Asymmetric) \\
\hline
\end{tabular}




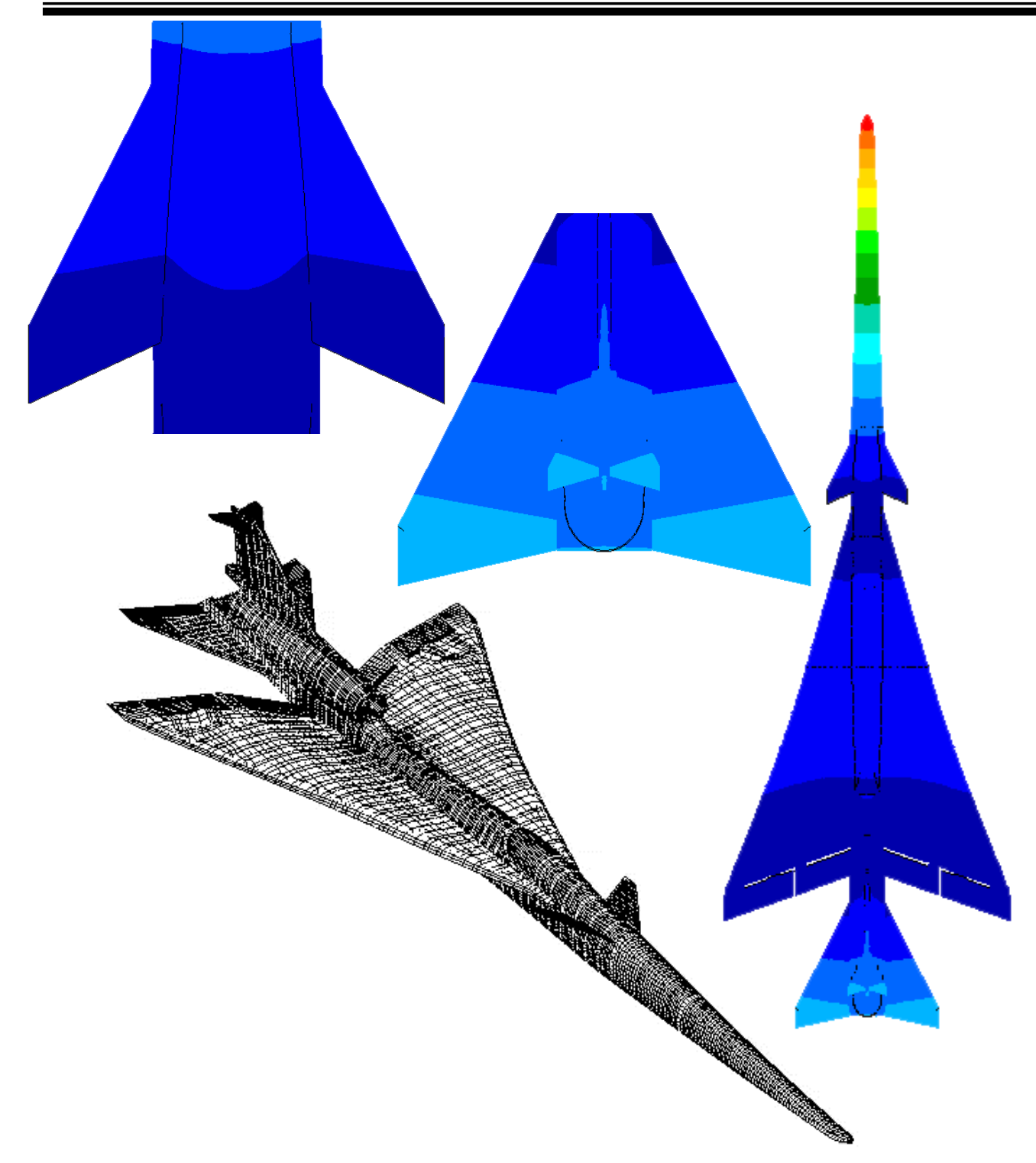

first fuselage vertical bending

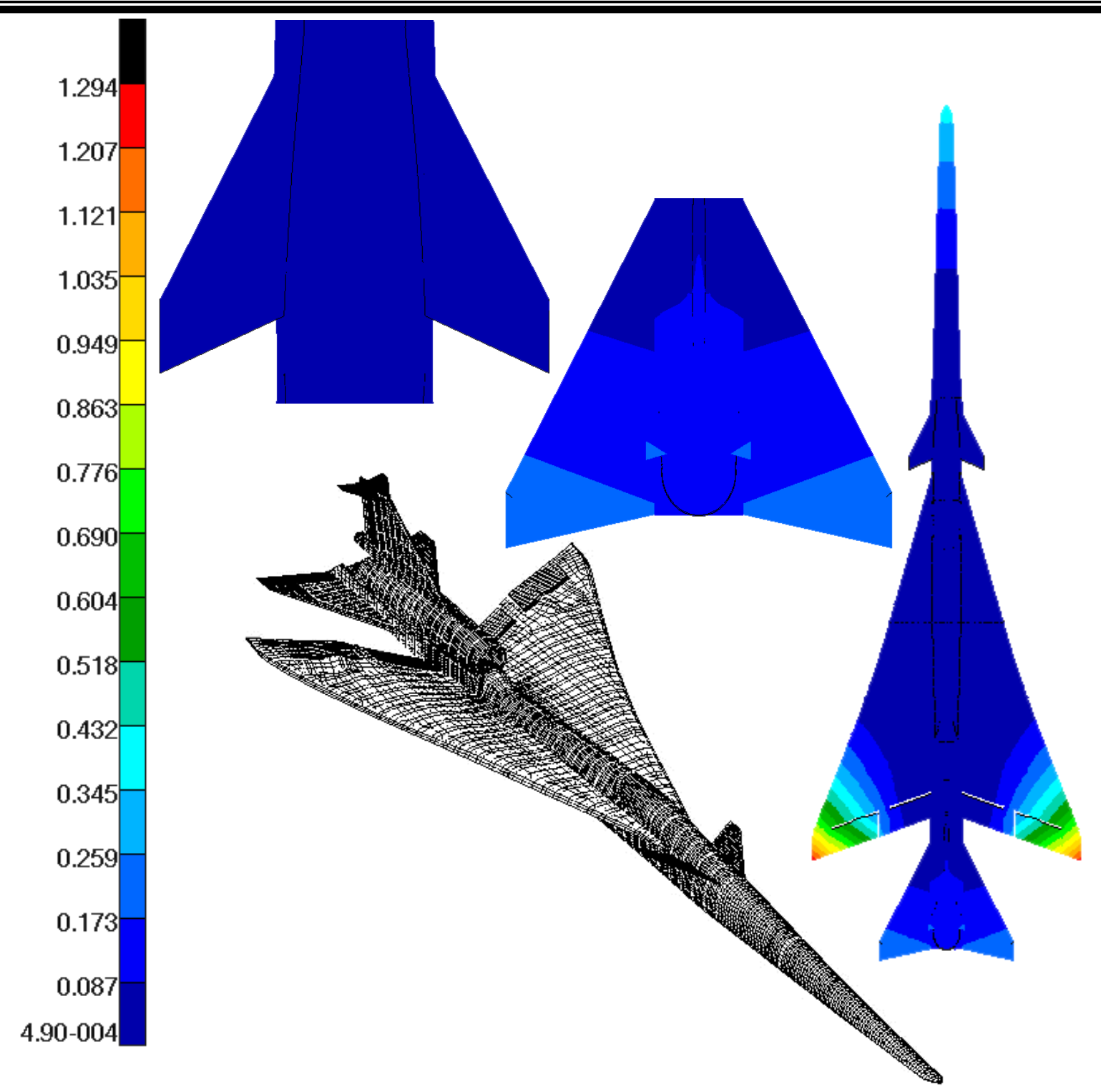

1.468
1.370
1.272
1.174
1.076
0.978
0.881
0.783
0.685
0.587
0.489
0.392
0.294
0.196
0.098
$2.74-004$

Symmetric first wing bending + forward fuselage vertical bending + horizontal tail rotation (in-phase: forward fuselage \& wing)(out-phase: wing and horizontal tail) 


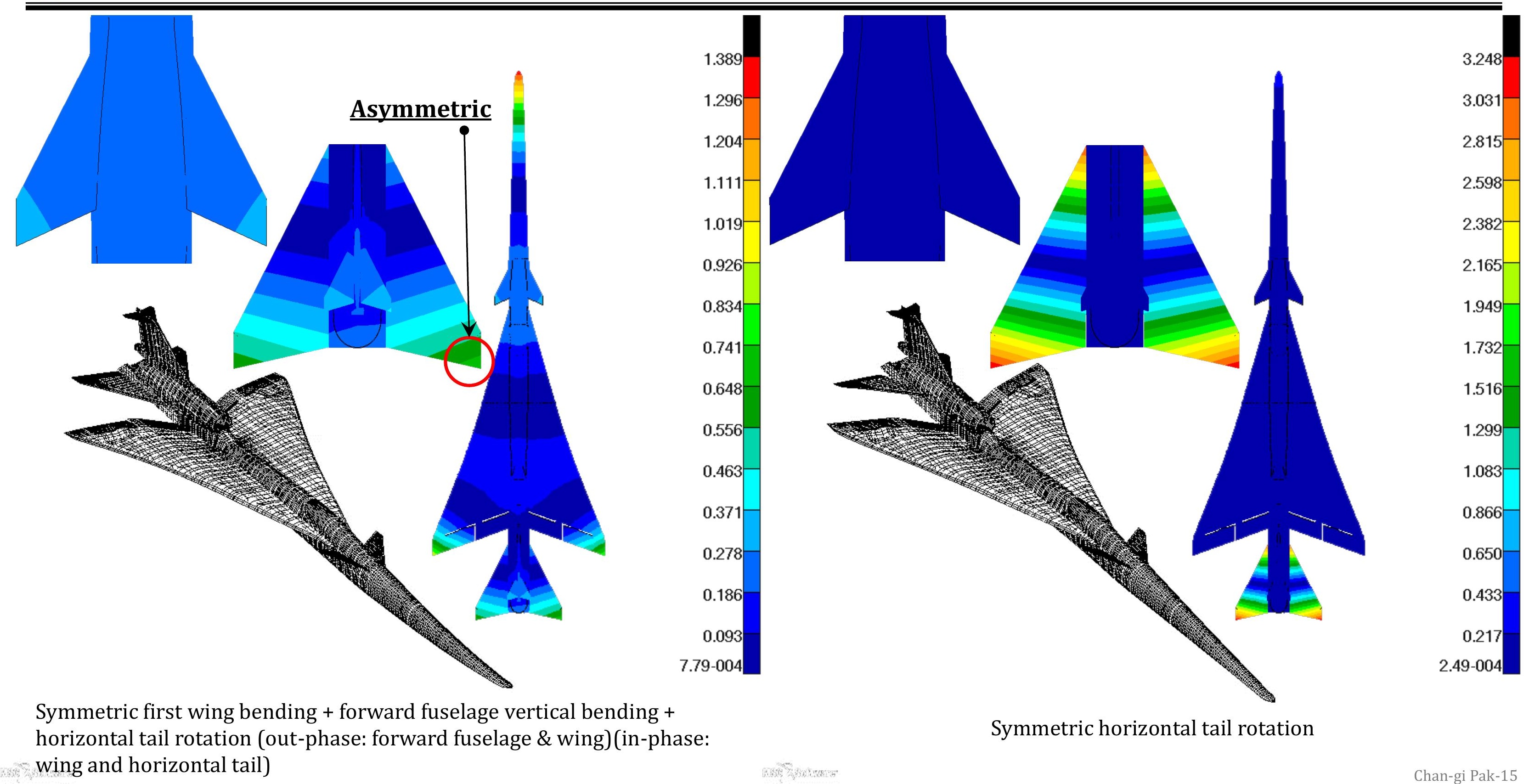



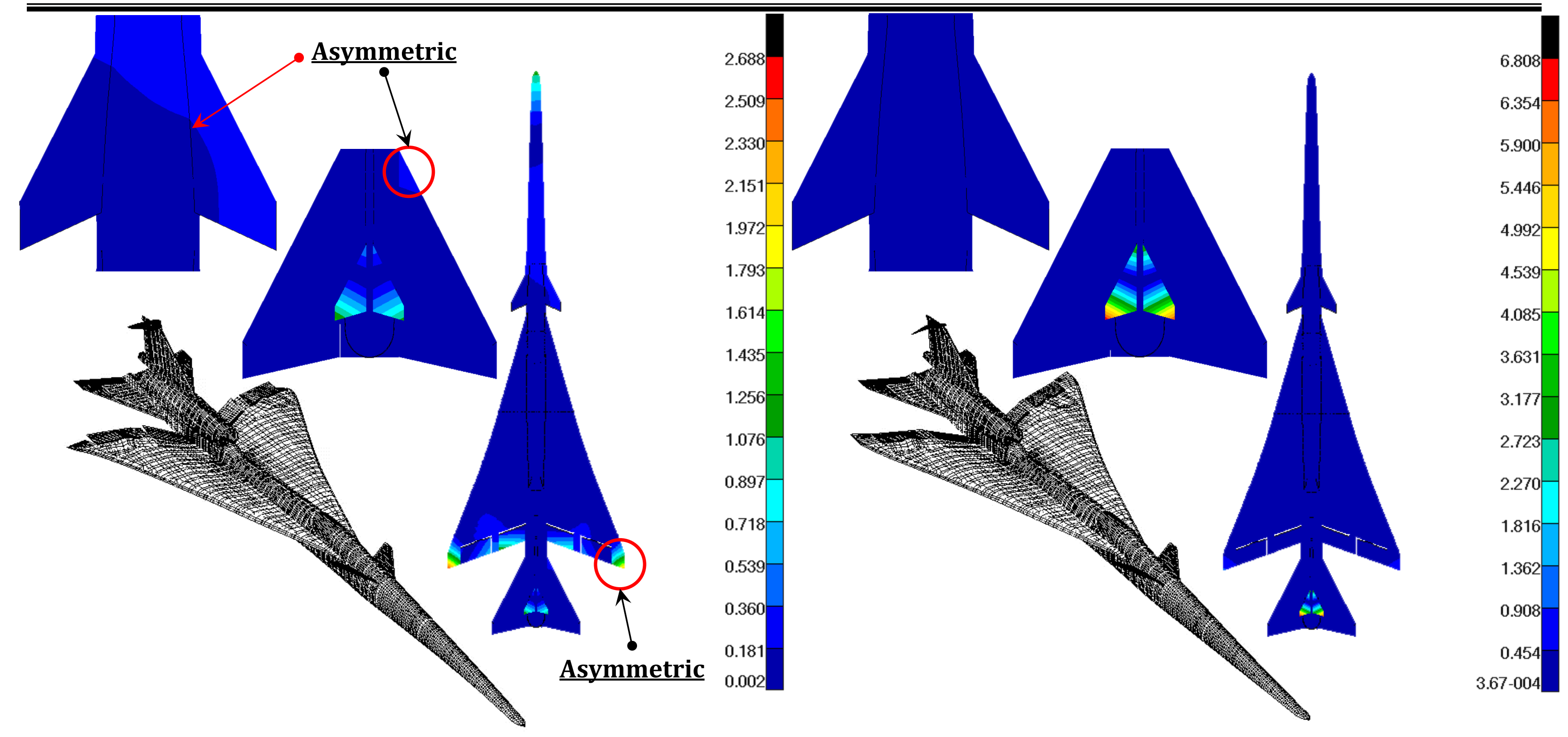


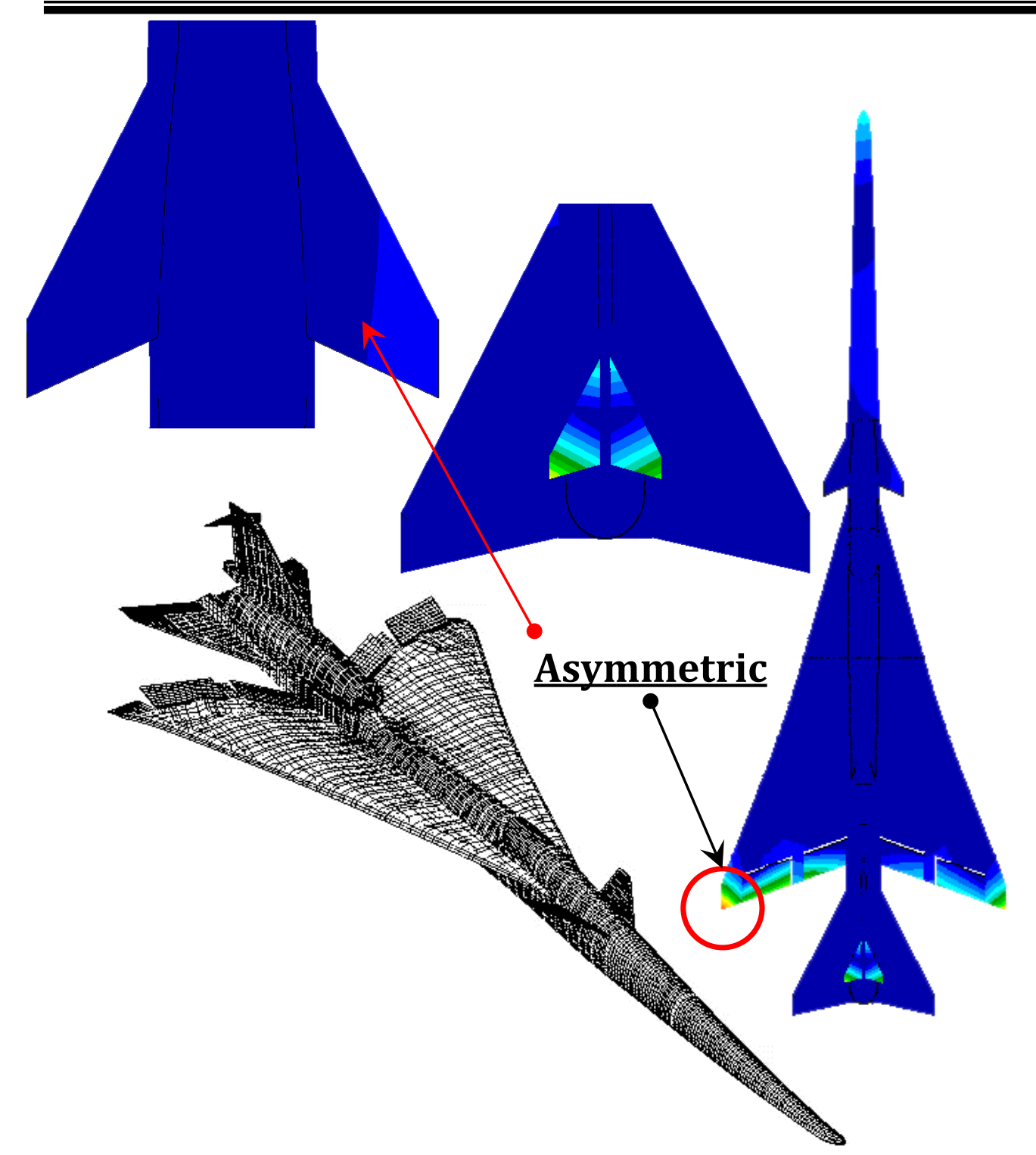

symmetric wing tip bending + ttail rotation flap \& airleron rotation + forward fuselage bending + nose landing gear vertical bending (out-phase wing tip \& forward fuselage) (out phase wing tip \& ttail)

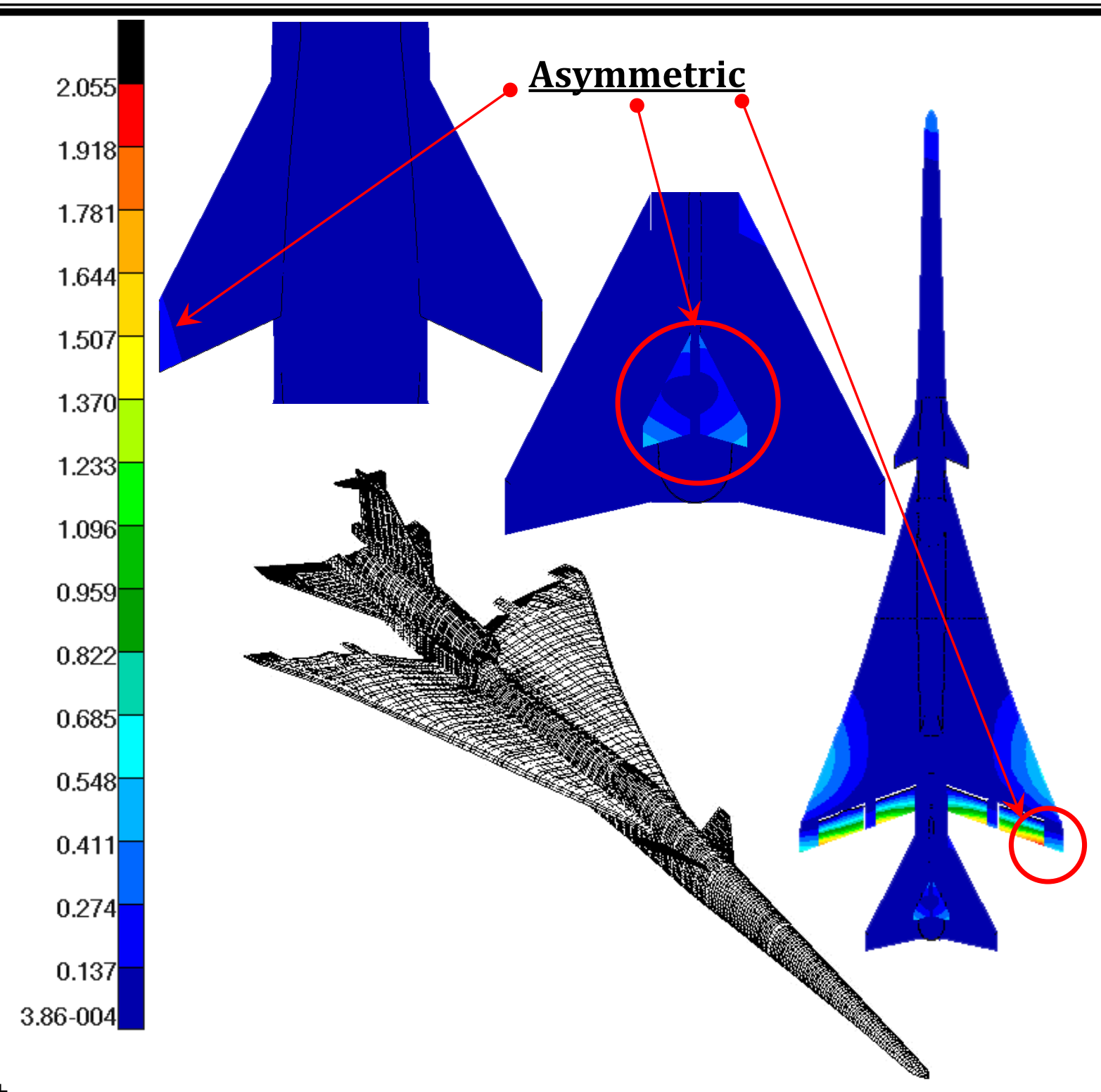

symmetric airleron + flaperon (in-phase)+ttail(pitch +yaw) 


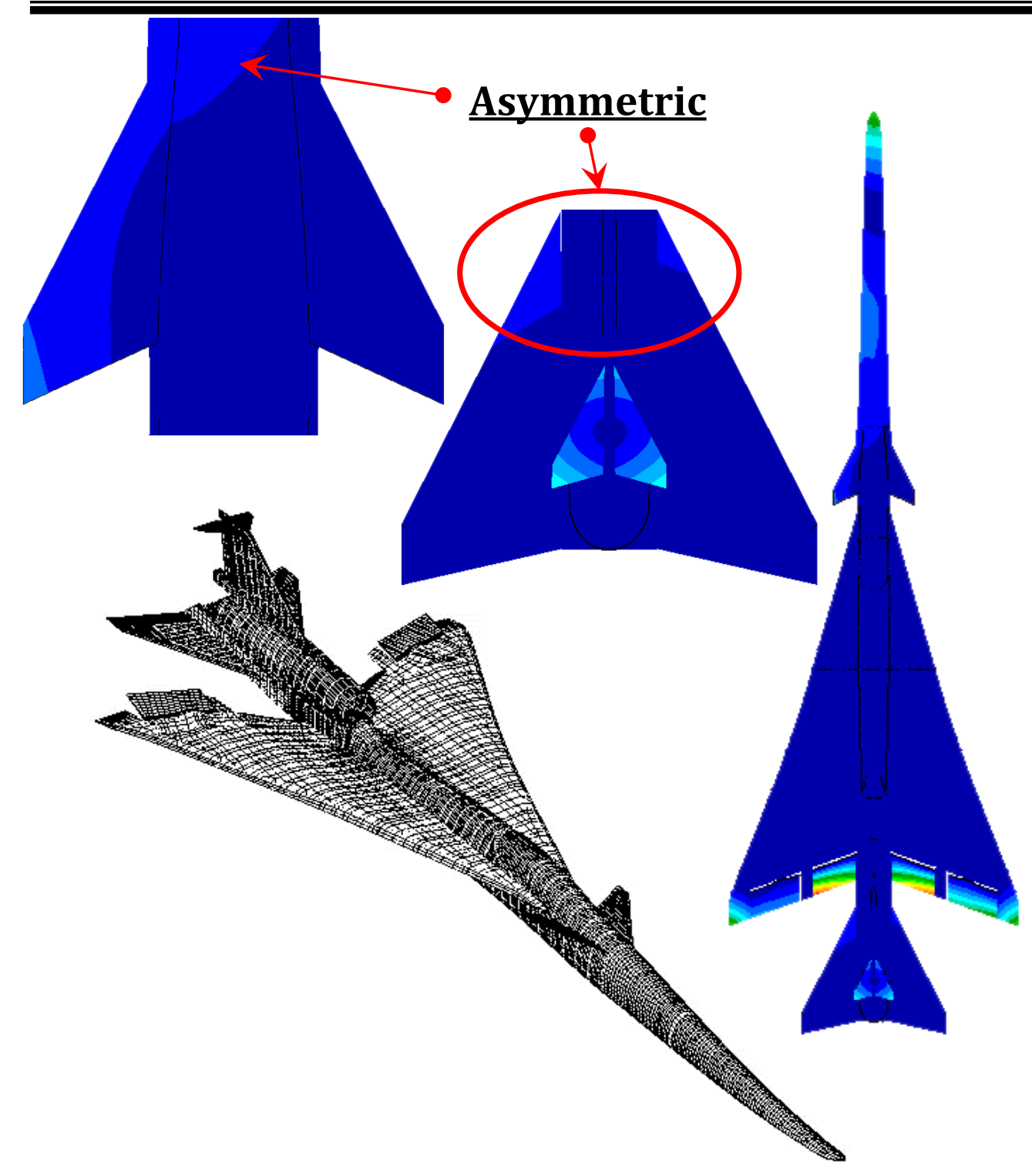

symmetric Flaperon+airleron (out-phase) +ttail(pitch+yaw) +forward fulage and airleron(in-phase)

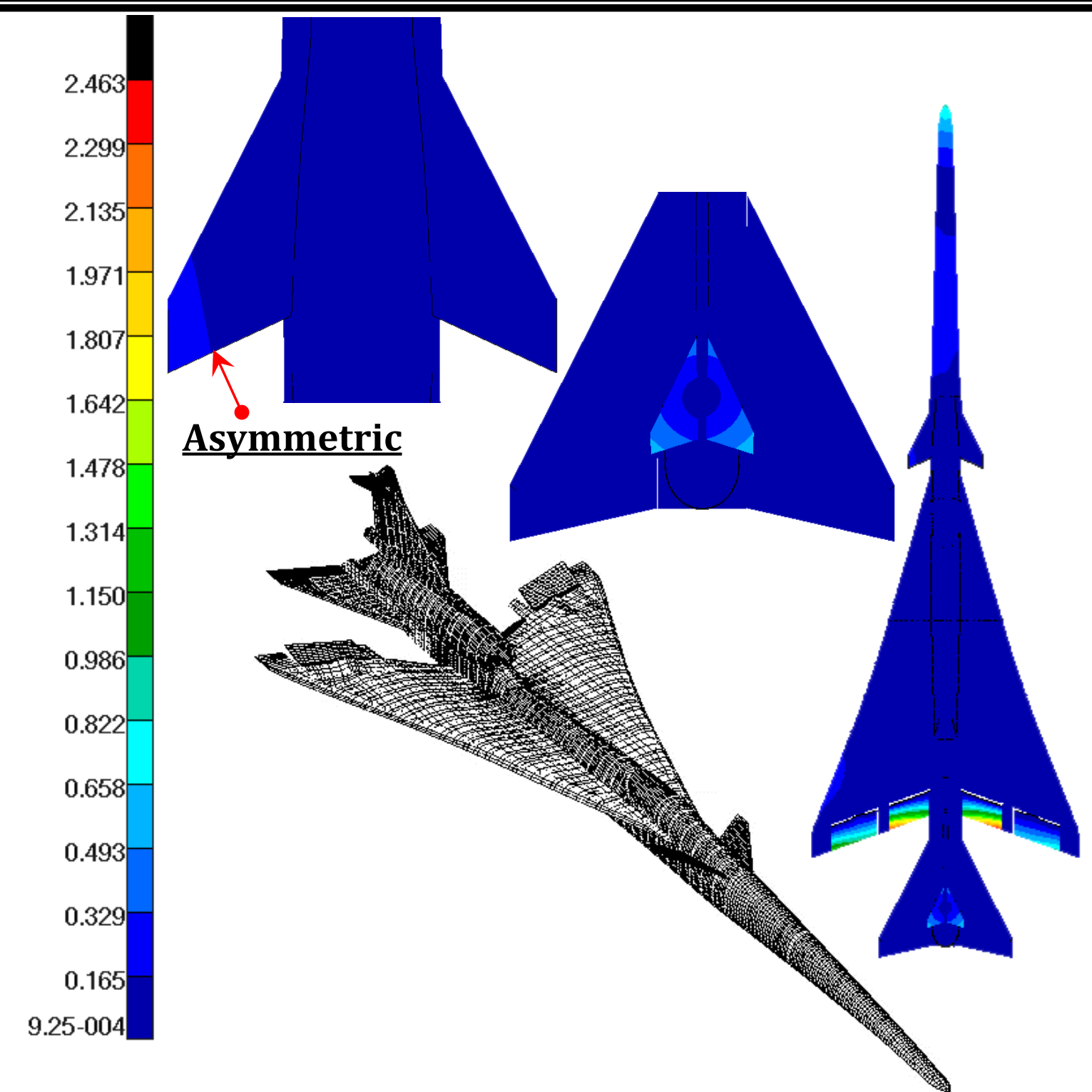

3.299
3.079
2.859
2.639
2.419
2.199
1.979
1.759
1.540
1.320
1.100
0.880
0.660
0.440
0.220
$4.92-004$

symmetric flaperon+airleron (out-phase)+ttail(pitch+yaw) + forward fulage and airleron(out-phase) 


\section{Trim Shape Difference (Baseline Configuration)}

口 Weight:

* Cruise $=18500.00 \mathrm{lbf}$

- Forward CG location

* $\mathrm{x}=836.09$ inch, $\mathrm{y}=-\mathbf{0 . 1 8 9 7}$ inch, $\mathrm{z}=100.68$ inch

(] Mach: 1.42

ㄱ Altitude: $55000 \mathrm{ft}$

- Aileron deflection angle: $0.0 \mathrm{deg}$

T-tail deflection angle: $0.0 \mathrm{deg}$

$\square\{\Delta T\}_{t o} \equiv\{T\}_{t}-\{T\}_{o}$

* $\{T\}_{t}=$ target trim shape at surface GRIDs

- $\{T\}_{o}=$ trim shape based on optimum jig shape

$\checkmark\{j i g\}_{o} \equiv\{j i g\}_{b}+[\boldsymbol{\Phi}]\{X\}_{o}$

$\checkmark\{j i g\}_{o} \underset{\text { trim analysis }}{\longrightarrow}\{T\}_{o}$

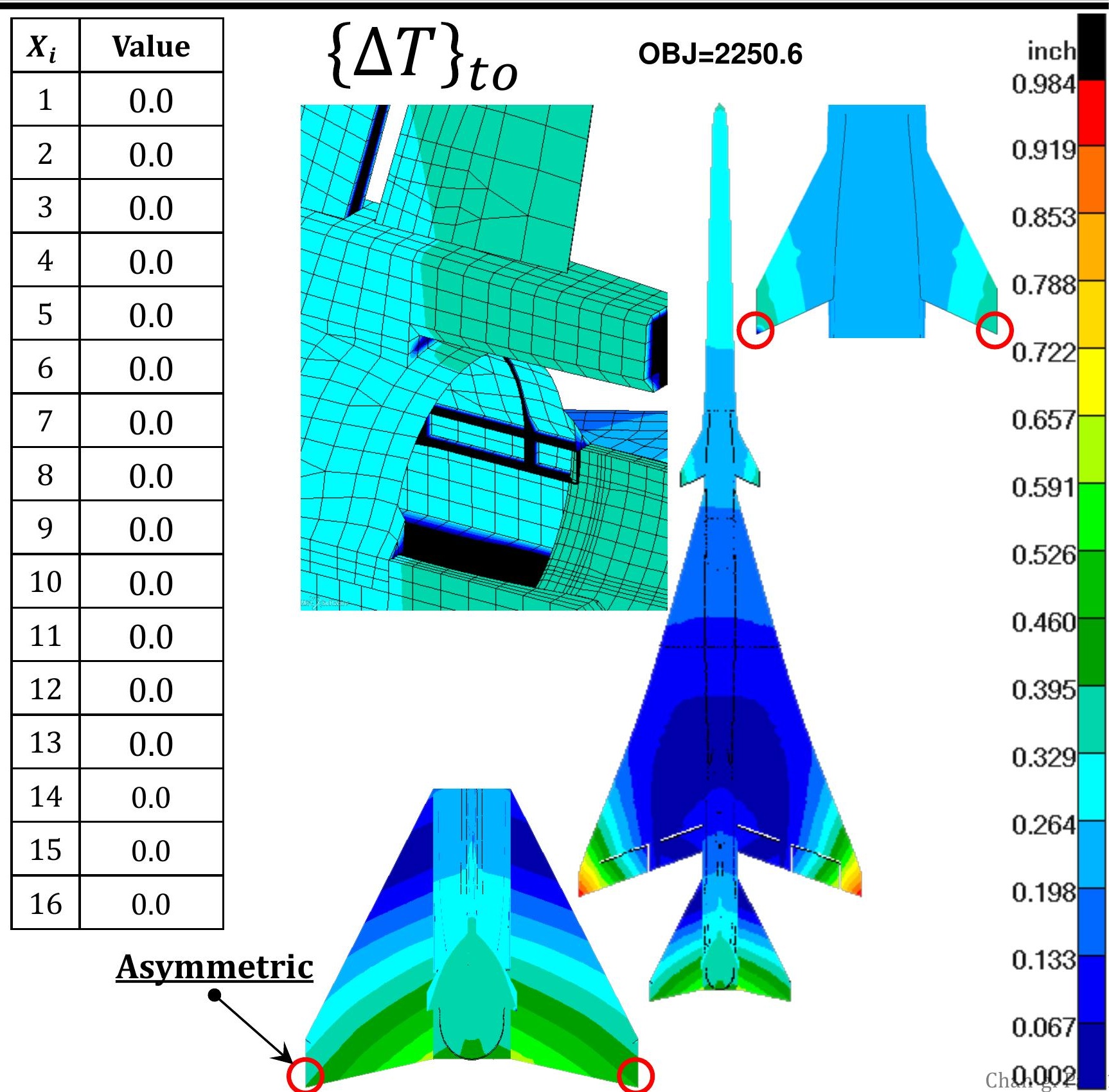




\section{Optimization \#2: $\{\Delta T\}_{t o}=\{T\}_{t}-\{T\}_{o}$}

\begin{tabular}{|c|c|}
\hline$X_{i}$ & Value \\
\hline 1 & -0.5783 \\
\hline 2 & -1.3482 \\
\hline 3 & -1.3008 \\
\hline 4 & 0.4226 \\
\hline 5 & -1.0585 \\
\hline 6 & 0.2544 \\
\hline 7 & 1.0823 \\
\hline 8 & .00555 \\
\hline 9 & 0.1242 \\
\hline 10 & .02061 \\
\hline 11 & -.04842 \\
\hline 12 & -02884 \\
\hline 13 & 0.1055 \\
\hline 14 & 0.2174 \\
\hline 15 & -.07665 \\
\hline 16 & \\
\hline
\end{tabular}

$O B J=6.255$
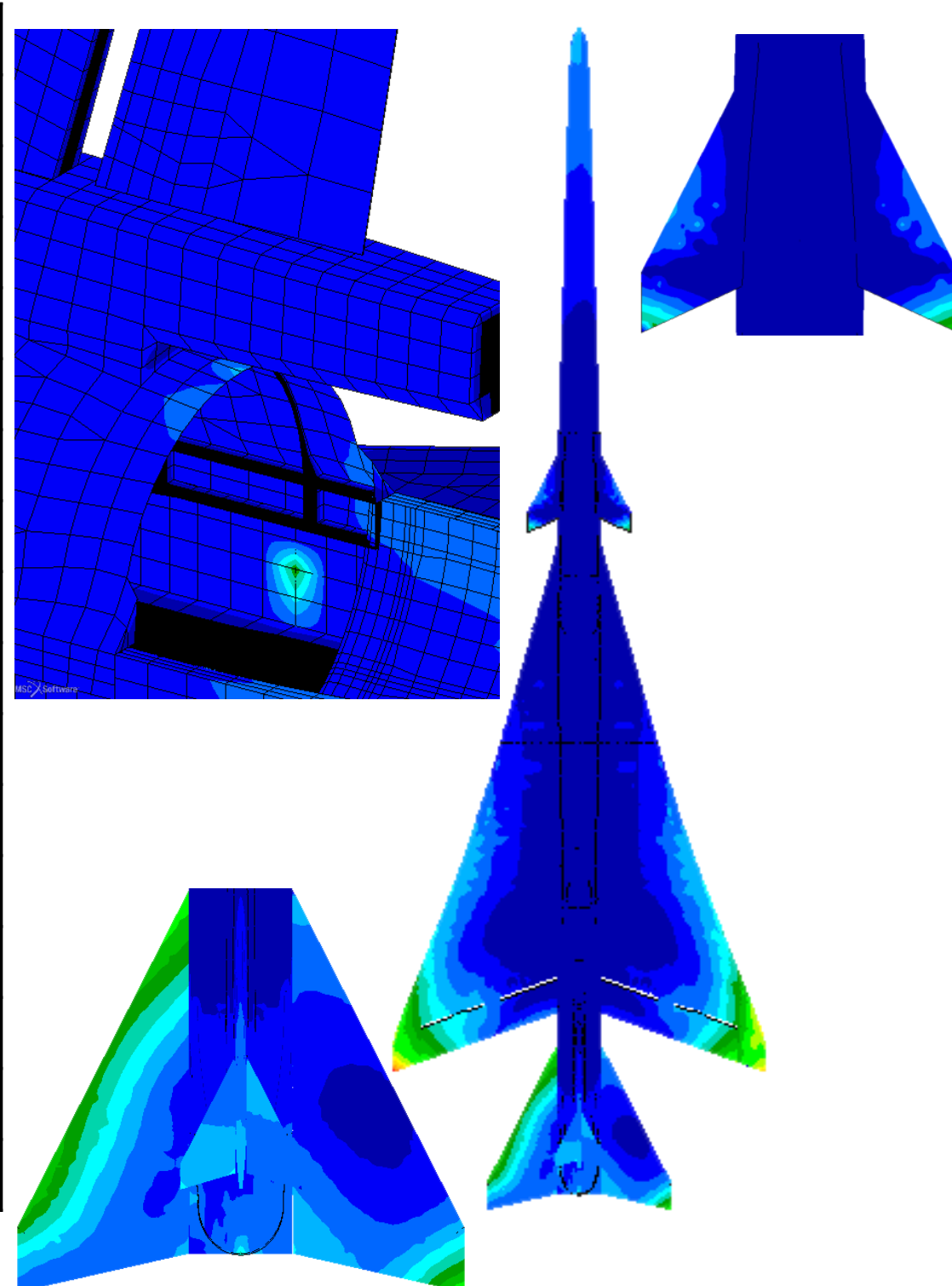

Start Configuration

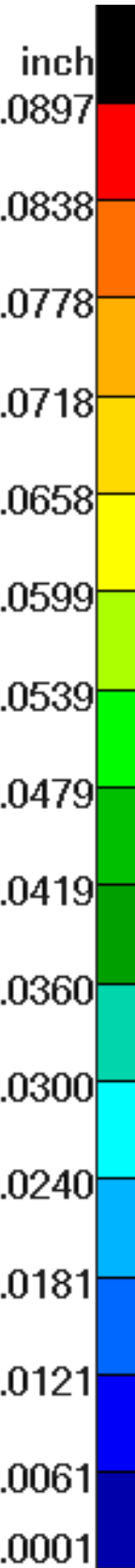

Use Optimization

\begin{tabular}{|c|c|}
\hline $\boldsymbol{X}_{\boldsymbol{i}}$ & Value \\
\hline 1 & -0.5759 \\
\hline 2 & -1.3489 \\
\hline
\end{tabular}

.0718

\begin{tabular}{|l|l|}
\hline 2 & -1.3489 \\
\hline 3 & -1.3013 \\
\hline 4 & 0.4228 \\
\hline
\end{tabular}

\begin{tabular}{|l|l|}
\hline 4 & 0.4228 \\
\hline 5 & -1.0587 \\
\hline 6 & 0.2541 \\
\hline 7 & \\
\hline
\end{tabular}

\begin{tabular}{|l|l|}
\hline 7 & 1.0814 \\
\hline 8 & .00513 \\
\hline
\end{tabular}

\begin{tabular}{|l|l|}
\hline 8 & .00513 \\
\hline 9 & 0.1243 \\
\hline
\end{tabular}

\begin{tabular}{|c|c|}
\hline 9 & 0.1243 \\
\hline 10 & .02058 \\
\hline
\end{tabular}

\begin{tabular}{|l|l|}
\hline 10 & .02058 \\
\hline 11 & -.04848 \\
\hline 12 & -.02907 \\
\hline 13 & 0.1056 \\
\hline 14 & 0.2172 \\
\hline 15 & -.07671 \\
\hline 16 & \multicolumn{2}{|}{} \\
\hline
\end{tabular}

.0001
OBJ $=6.232$

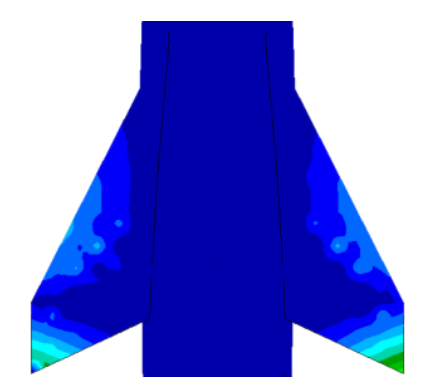

$\begin{array}{r}0664 \\ \hline\end{array}$

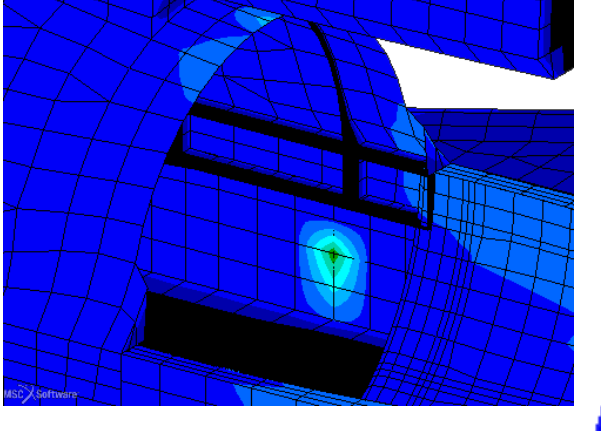

.0604

.0544

.0483

.0423

.0363

.0303

.0242

.0182

.0122

.0062

Optimum Configuration 


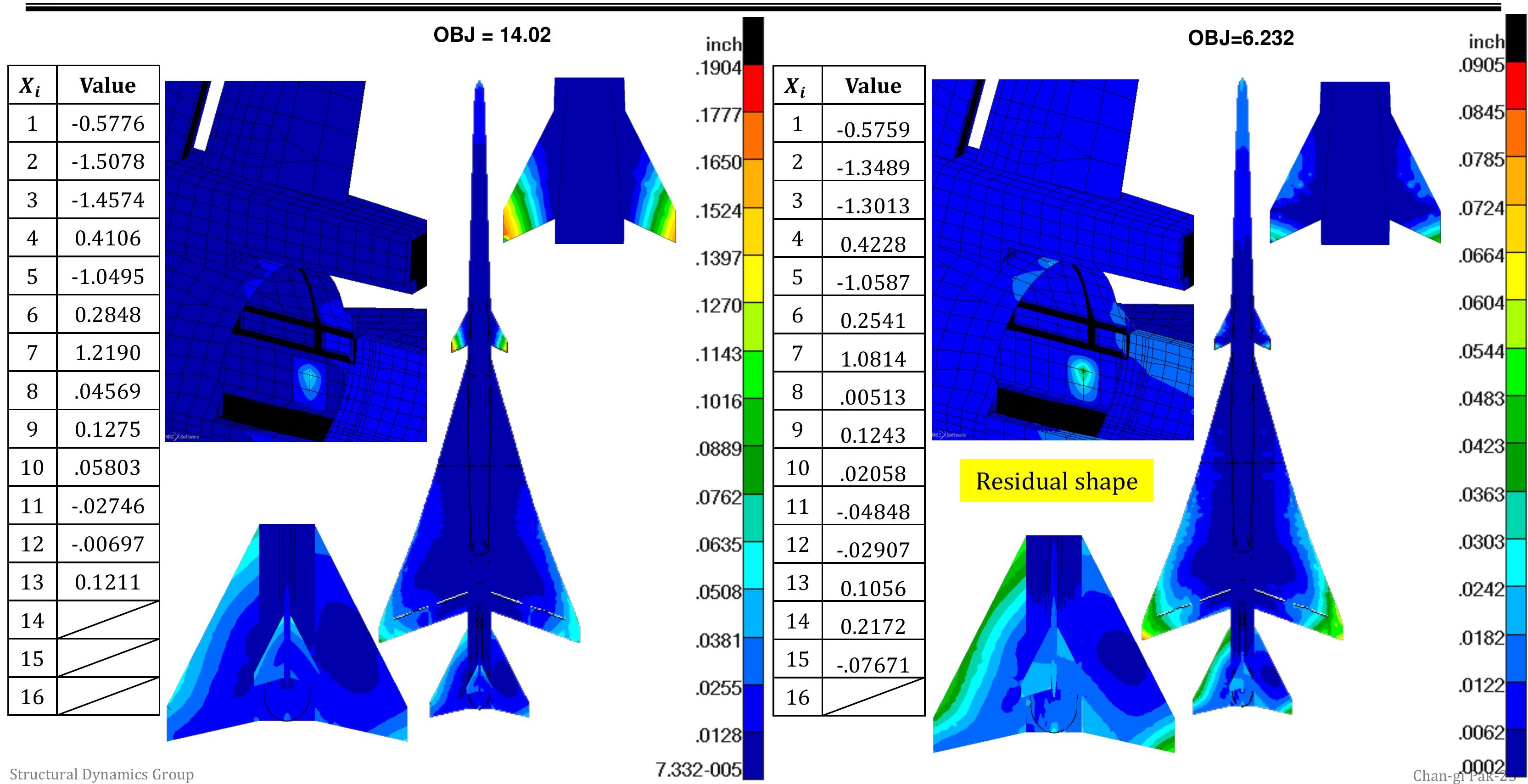




\section{Optimization \#3: $\{\Delta T\}_{t o}=\{T\}_{t}-\{T\}_{o}$}

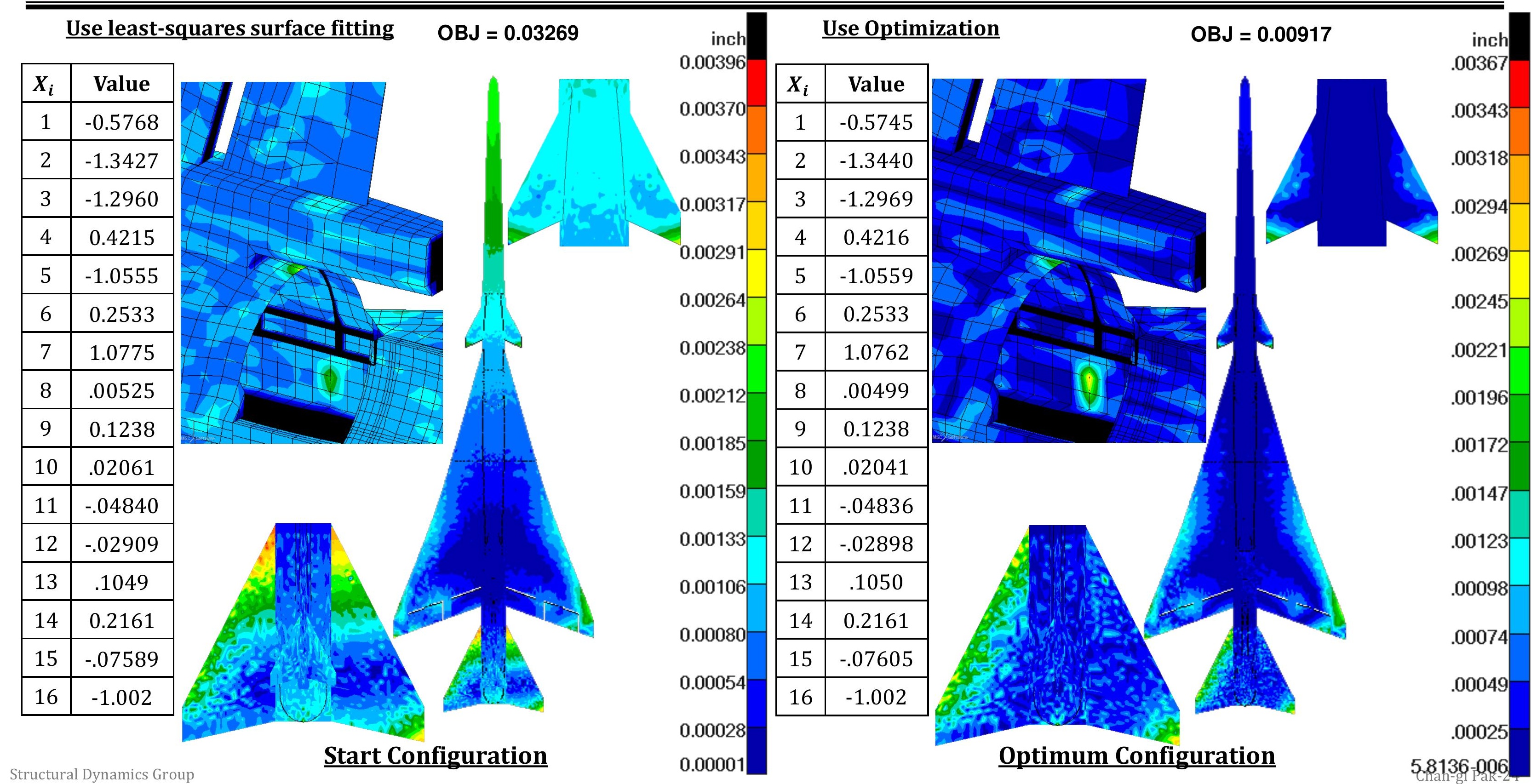


Optimization Results

\begin{tabular}{|c|c|c|c|c|c|c|c|c|}
\hline \multirow{2}{*}{ DESVAR ID } & \multirow{2}{*}{ Baseline } & \multicolumn{2}{|c|}{ Optimization \#1 } & \multicolumn{2}{|c|}{ Optimization \#2 } & \multicolumn{2}{|c|}{ Optimization \#3 } & \multirow{2}{*}{ Comments } \\
\hline & & Start & Optimum & Start & Optimum & Start & Optimum & \\
\hline 1 & 0.0 & -0.5795 & -0.5776 & -0.5783 & -0.5759 & -0.5768 & -0.5745 & Rigid pitch \\
\hline 2 & 0.0 & -1.5063 & -1.5078 & -1.3482 & -1.3489 & -1.3427 & -1.3440 & Stabilator_R \\
\hline 3 & 0.0 & -1.4565 & -1.4574 & -1.3008 & -1.3013 & -1.2960 & -1.2969 & Stabilator_L \\
\hline 4 & 0.0 & 0.4108 & 0.4106 & 0.4226 & 0.4228 & 0.4215 & 0.4216 & Mode 7 \\
\hline 5 & 0.0 & -1.0492 & -1.0495 & -1.0585 & -1.0587 & -1.0555 & -1.0559 & Mode 9 \\
\hline 6 & 0.0 & 0.2851 & 0.2848 & 0.2544 & 0.2541 & 0.2533 & 0.2533 & Mode 11 \\
\hline 7 & 0.0 & 1.2202 & 1.2190 & 1.0823 & 1.0814 & 1.0775 & 1.0762 & Mode 15 \\
\hline 8 & 0.0 & .04660 & .04569 & .00555 & .00513 & .00525 & .00499 & Mode 17 \\
\hline 9 & 0.0 & 0.1273 & 0.1275 & 0.1242 & 0.1243 & 0.1238 & 0.1238 & Mode 19 \\
\hline 10 & 0.0 & .05808 & .05803 & .02061 & .02058 & .02061 & .02041 & Mode 20 \\
\hline 11 & 0.0 & -.02754 & -.02746 & -.04842 & -.04848 & -.04840 & -.04836 & Mode 22 \\
\hline 12 & 0.0 & -.00712 & -.00697 & -.02884 & -.02907 & -.02909 & -.02898 & Mode 23 \\
\hline 13 & 0.0 & 0.1212 & 0.1211 & 0.1055 & 0.1056 & 0.1049 & .1050 & Mode 25 \\
\hline 14 & 0.0 & & & 0.2174 & 0.2172 & 0.2161 & 0.2161 & Mode 37 \\
\hline 15 & 0.0 & & & -.07665 & -.07671 & -.07589 & -.07605 & Mode 48 \\
\hline 16 & 0.0 & & & & & -1.002 & -1.002 & Residual \\
\hline Maximum Error (inch) & 0.9844 & 0.1896 & 0.1904 & .0897 & .0905 & .00396 & .00367 & \\
\hline Objective Function & 2250.6 & 14.04 & 14.02 & 6.255 & 6.232 & .03269 & .00917 & \\
\hline
\end{tabular}

年




\section{Optimum Jig-Shape Configuration with rigid rotation modes}

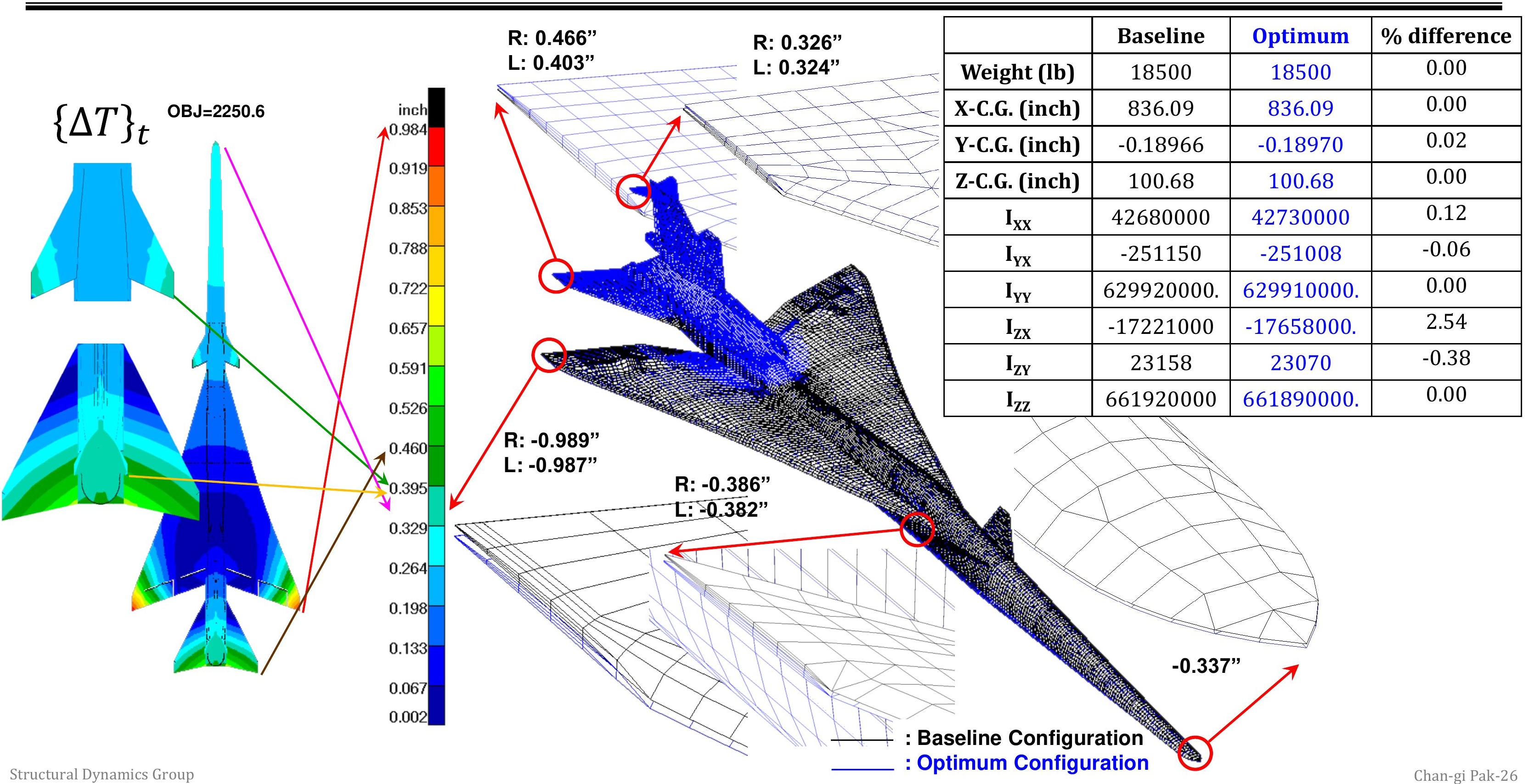




\section{Optimum Jig-Shape Configuration without rigid rotation modes}

\section{R: 0.181 "'}

L: 0.152"

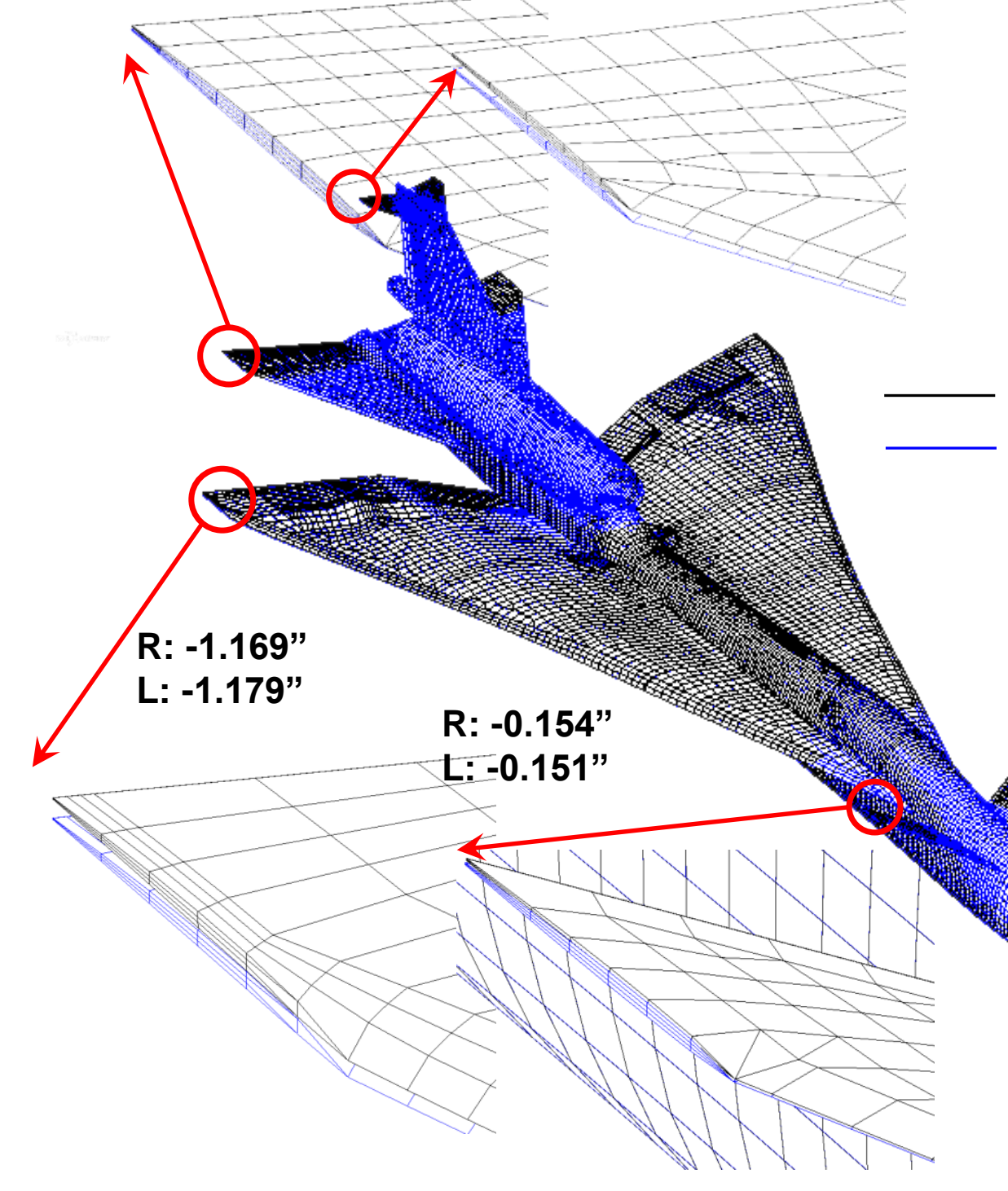

R: -0.463 "

R: -0.463
Structural assumption

Baseline (degree)

Optimum (degree)

Rigid

Flexible

.



3.0

3.0624

3.1036

: Baseline Configuration : Optimum Configuration

$\square$ Baseline rigid AOA - Optimum flexible AOA

$$
\text { * } 3.0624 \mathrm{deg}-3.1033 \mathrm{deg}=-0.0409 \mathrm{deg}=-0.000713 \mathrm{rad}
$$

$\square$ Distance from fuselage nose to center of gravity location * $(25.46,0.0,82.51)-(836.09,-0.1897,100.68)=810.83$ inch

$\square \mathrm{Z}$ deflection due to AOA difference * $810.83 \times \tan (-0.000713)=-0.579$ inch $(-0.575$ inch $)$

$\square$ Flexible AOA effect at the fuselage nose * 0.240 inch -0.579 inch $=-0.339$ inch $(-0.337$ inch $)$ 


\section{Summary of Natural Frequencies before and after optimization}

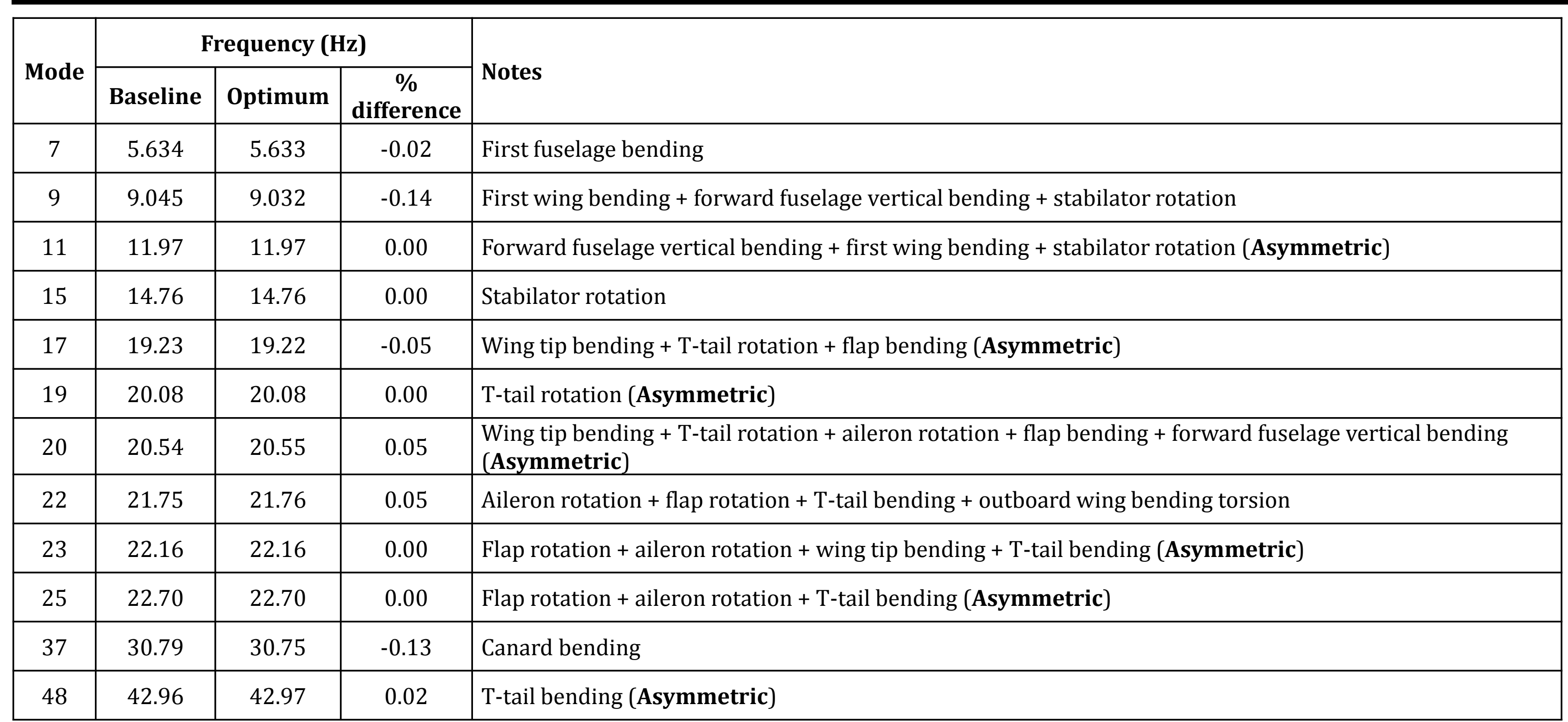


In this study, the jig-shape optimization is performed using the two step approach.

* The first step is computing the starting design variables using the least squares surface fitting technique.

* The next step is the fine tune of the jig-shape using the numerical optimization procedure.

* Assume unconstrained optimization

The maximum frequency change due to the jig-shape optimization is less than $0.14 \%$.

$>$ The minor changes in mass moment of inertia are observed. (mostly less than $0.38 \%$; maximum $2.54 \%$ )

Sixteen basis function are used in this jig-shape optimization study.

* Total of twelve symmetric mode shapes of the cruise weight configuration. (Asymmetric shapes exist)

Fitting trim deformation

* Three basis functions for trim variables (rigid pitch shape, rigid left and right stabilator rotation shapes)

$>$ Fitting flexibility effect on trim variables

* A residual shape is also selected as a basis function.

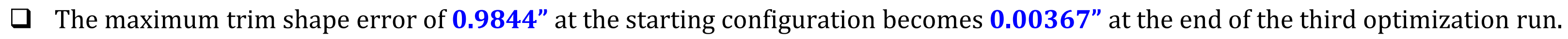




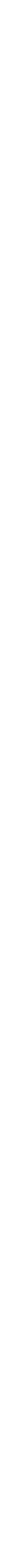

